\title{
Caractérisation des principaux miels des régions du Nord de l'Algérie*
}

\author{
Djamila Benaziza-Bouchema ${ }^{1}$ \\ Paul Schweitzer ${ }^{2}$ \\ ${ }^{1}$ École nationale supérieure agronomique \\ Département des productions animales \\ Hacen Badi \\ 16200 El Harrach \\ 16200 Alger \\ Algérie \\ <benaziza_djamila@yahoo.fr> \\ ${ }^{2}$ Centre d'études techniques apicole \\ de Moselle (Cetam) \\ Laboratoire d'analyse et d'écologie apicole \\ $1 \mathrm{a}$, rue Jean-Baptiste de la Salle \\ 57310 Guénange \\ <cetam@club-internet.fr>
}

\begin{abstract}
Résumé
Les propriétés physico-chimiques (teneur en eau, pH, conductivité électrique, acidité totale, hydroxyméthylfurfural, activité diastasique, taux de glucose, de fructose et de saccharose) ainsi que l'analyse pollinique (palynologie) ont été utilisées afin de caractériser 28 échantillons de miels du Nord de l'Algérie. Les résultats des analyses polliniques démontrent la dominance des miels monofloraux de Citrus et d'Eucalyptus, avec des pourcentages de pollen variant respectivement de 50 à $60 \%$ et de 80 à $99 \%$, ainsi que des miels polyfloraux. Ces différents miels caractérisent les régions d'Alger, de Blida, de Tipaza et de Boumerdès. La teneur en eau, le pH, la conductivité électrique, l'acidité totale, l'hydroxyméthylfurfural et l'activité diastasique sont inférieurs ou égaux à 18,6\% $\pm 0,28,4,23 \pm 0,01,0,80 \pm 0,06 \mathrm{~ms} / \mathrm{cm}, 50,4 \mathrm{meq} / \mathrm{kg}, 38,7 \pm 1,8 \mathrm{mg} / \mathrm{kg}$ et $34 \pm 1,2$ unités de Schade/g respectivement. Les taux de glucose, de fructose et de saccharose présentent des teneurs respectives de 37,4\% $1,02,45,9 \% \pm 0,78$ et 7,6\% 0,08 comme limites supérieures. Ces résultats ont montré que ces miels répondent aux normes internationales et leur caractérisation permettra d'obtenir des labels de qualité propres à l'Algérie.
\end{abstract}

Mots clés : abeille domestique ; Algérie ; label de qualité ; miel ; palynologie.

Thèmes : productions végétales ; qualité et sécurité des produits.

\section{Abstract \\ Characterization of the main honeys from the northern regions of Algeria}

The physico-chemical properties (moisture content, $\mathrm{pH}$, electric conductivity, total acidity, hydroxymethylfurfural (HMF), diastasic activity, contents in glucose, fructose and saccharose) as well as pollen analysis (palynology) were used to characterize 28 honey samples from the North of Algeria. Pollen analysis results showed the dominance of monofloral honeys from Citrus and Eucalyptus with percentages of pollen varying respectively from 50 to $60 \%$ and 80 to $99 \%$ as well as polyfloral honeys. These honeys are characteristic of the regions of Algiers, Blida, Tipaza and Boumerdès. Moisture content, $\mathrm{pH}$, electric conductivity, total acidity, hydroxymethylfurfural and diastasic activity were lower or equal to $18.6 \% \pm 0.28,4.23 \pm 0.01,0.80 \pm 0.06 \mathrm{~ms} / \mathrm{cm}, 50.4 \mathrm{meq} / \mathrm{kg}$, $38.7 \pm 1.8 \mathrm{mg} / \mathrm{kg}$ et $34 \pm 1.2$ units of Schade/g, respectively. The results of physicochemical analysis showed that these honeys met international standards and their characterization will make it possible for Algeria to obtain quality labels.

Key words: honey; honey bees; palynology; quality labels.

Subjects: vegetal productions; product quality and security. n Algérie, le secteur de l'agriculture a mis en place durant l'année 2000 une stratégie opérationnelle de développement agricole (plan national de développement agricole, PNDA) élargie, à partir de 2002, au domaine rural à la

* Pour citer cet article: Benaziza-Bouchema D, Schweitzer P. Caractérisation des principaux miels des régions du Nord de l'Algérie. Cah Agric 2010 ; 19 : 432-8. doi : 10.1684/agr.2010.0432 
faveur de nouvelles attributions confiées par le Gouvernement au ministère de l'Agriculture et du Développement rural. Dans ce contexte, une attention a été donnée aux productions apicoles et en particulier à la mise en place de ruches modernes et à la production de miel. Notre travail a pour but l'identification des principaux miels monofloraux des régions du Nord de l'Algérie et de vérifier la qualité ainsi que l'appellation des miels par l'utilisation des analyses physico-chimiques et palynologiques. L'analyse des pollens du miel ou mélisso-palynologie est de la plus grande importance pour le contrôle de la qualité du miel (Mateo et Bosch-Reig, 1997 ; Anklam, 1998; Anklam et Radovic, 2001 ; Popek, 2002 ; Terrab et al., 2002 ; Devilliers et al., 2004). Selon Louveaux (1985), le problème de la détermination de l'origine botanique des miels est trop complexe pour être, dans tous les cas, résolu par l'utilisation d'un seul critère. L'analyse pollinique associée à de nombreux éléments d'ordre physicochimique permet d'émettre sur l'origine botanique un jugement d'ensemble valable. La caractérisation de l'appellation monofloral est fondée à la fois sur des analyses physico-chimiques et polliniques. En général, les miels monofloraux possèdent un ou plusieurs critères physico-chimiques discriminants qui vont les différencier des autres miels monofloraux et des miels polyfloraux. L'objectif de cette recherche est l'établissement des caractéristiques physico-chimiques et palynologiques des principaux types de miels des régions du Nord de l'Algérie.

\section{Matériel et méthode}

\section{Les échantillons de miel}

Notre étude a porté sur 28 échantillons de miels provenant des principales régions mellifères de la plaine de la Mitidja située au centre de l'Algérie du Nord, à savoir les préfectures d'Alger $\left(36^{\circ} 45^{\prime} 0^{\prime \prime} \mathrm{N}\right.$ et $3^{\circ} 10^{\prime}$ 0" E) (échantillons A1 à A7), Blida (36 34' 59" N et $3^{\circ} 0^{\circ} 0^{\prime}$ "E) (échantillons Ba1 à Ba7), Tipaza (36 34' $59^{\prime \prime}$ N et $2^{\circ} 25^{\prime}$ $0^{\prime}$ 'E) (échantillons T1 à T7) et Boumerdès (36 45' $6^{\circ} " \mathrm{~N}$ et $3^{\circ} 40^{\prime} 0^{\prime \prime} \mathrm{E}$ ) (échantillons Bs1 à Bs7).

Les miels ont été récoltés par les apiculteurs entre le mois de mai et le mois de juillet 2006. Après extraction électrique du miel des cadres, les échantillons sont homogénéisés et mis dans des récipients hermétiques de $1 \mathrm{~kg}$. La durée de stockage est de 15 jours pour les analyses physicochimiques et de 1 mois pour les analyses palynologiques. Toutes les analyses ont été effectuées en trois répétitions.

\section{Présentation de la zone d'étude}

La Mitidja est une vaste plaine alluviale du Nord algérien (figure 1). C'est une dépression longue d'environ $100 \mathrm{~km}$ sur 15 à $20 \mathrm{~km}$ de large resserrée entre l'Atlas blidéen au sud et le Sahel au nord, largement ouverte sur la mer avec une superficie totale de $1400 \mathrm{~km}^{2}$ et une superficie agricole de 120000 à 130000 hectares. La diversité des sols présente des aptitudes très variées en matière de cultures : les agrumes sont principalement cultivés dans le centre de la plaine ainsi que le blé associé à des cultures fourragères et maraîchères. On y trouve également des cultures industrielles et de l'Eucalyptus.

Cette région dispose de potentialités mellifères et nectarifères appréciables qui lui permettent d'assurer un développement considérable de la production apicole.

\section{Analyses physico-chimiques des miels}

La conductivité électrique au 1/5 a été déterminée selon la méthode de Bogdanov (2002) en utilisant un conductimètre de type Knick model. Les mesures sont effectuées à $20^{\circ} \mathrm{C}$ dans une solution aqueuse à $20 \%$ par rapport à la matière sèche du miel. La lecture est faite directe- ment après immersion de la cellule dans la solution. Les résultats sont exprimés en milliSiemens par centimètre $(\mathrm{mS} / \mathrm{cm})$.

Le $\mathrm{pH}$ est mesuré à l'aide d'un pH-mètre de type HI 9025 - HANNA sur une solution de miel à $10 \%$ dans l'eau distillée. Leur teneur en eau est déterminée par la mesure de l'indice de réfraction à $20^{\circ} \mathrm{C}$ à l'aide d'un réfractomètre de type Abbé (RF 490, Euromexholland). Les indices de réfraction sont convertis selon la table de Chataway en teneur en eau selon la méthode harmonisée du miel développée par la Commission internationale du miel (Bogdanov, 2002). L'acidité totale est la somme de l'acidité libre et de l'acidité liée (des lactones); elle est exprimée en milliéquivalents d'acide pour $1000 \mathrm{~g}$ de miel. Le matériel utilisé lors de ce dosage est un titrateur automatique de type ORION. La quantité d'hydroxyméthylfurfural (HMF) a été mesurée par la méthode Winkler, dans une solution de miel à $20 \%$, méthode harmonisée de la Commission européenne du miel décrite par Bogdanov et al. (1997) dont le principe est fondé sur l'absorption dans le visible à $550 \mathrm{~nm}$ de la coloration obtenue après addition de $\rho$-toluidine et d'acide barbiturique. Les résultats obtenus sont exprimés en mg/kg. L'analyse a été effectuée en utilisant un spectrophotomètre UV-visible Cary 50.

L'activité diastasique a été déterminée par la méthode Phadebas, méthode harmonisée de la Commission européenne (Bogdanov et al., 1997) qui consiste en la mesure de la coloration bleue de l'amidon restant suite à une hydrolyse par la diastase à $40^{\circ} \mathrm{C}$ (bain thermostatique).

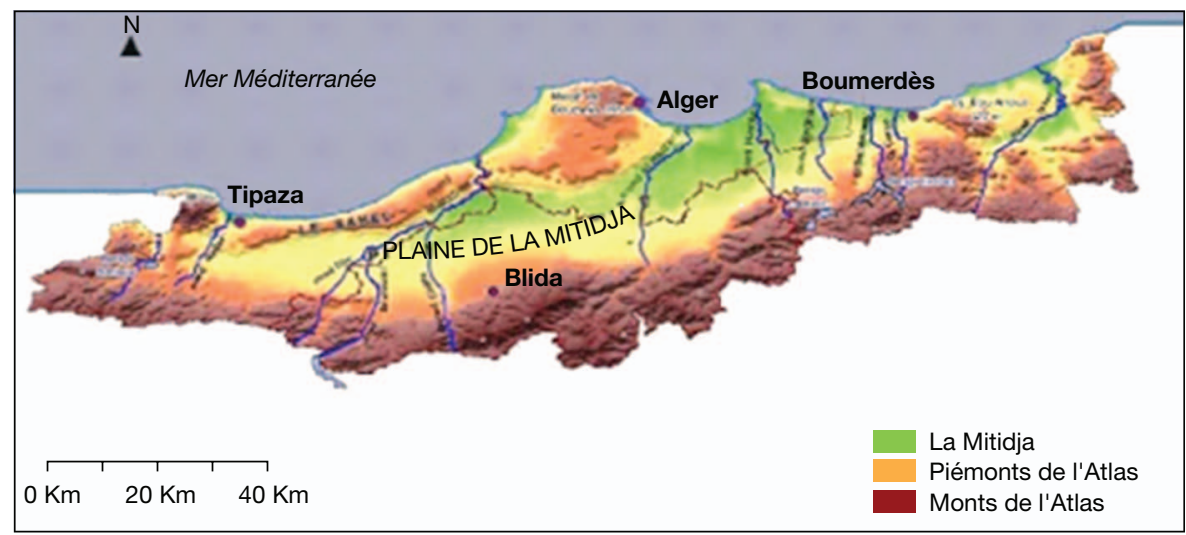

Figure 1. Présentation de la zone d'étude.

Figure 1. Presentation of the study zone.

Source : Programme d'aménagement côtier (PAC), 2006. 
Cette coloration est mesurée par spectrophotométrie à $620 \mathrm{~nm}$ (spectrophotomètre UV-visible Cary 50). L'absorbance de la solution est directement proportionnelle à l'activité diastasique de l'échantillon de miel. Les résultats sont exprimés en unités de Schade par gramme de miel. Le dosage des sucres est réalisé par chromatographie liquide à haute performance (HPLC) à détection ampérométrique pulsée selon la méthode de Bogdanov et al. (1997) en utilisant un chromatographe avec détecteur Coulochem équipé d'un module de dégazage (Hélium), d'une colonne Carbopac-AS6 (250x4 mm), d'une boucle d'injection de $25 \mu$ et d'un détecteur ampérométrique pulsé avec électrode de mesure en or et d'un intégrateur (Shimadzu C-R5A Chromatopac). Le débit est de $0,5 \mathrm{~mL} / \mathrm{min}$ pendant $16 \mathrm{~min}$, puis $0,1 \mathrm{~mL} / \mathrm{min}$ pendant $5 \mathrm{~min}$, ensuite $1 \mathrm{~mL} / \mathrm{min}$ pendant $16 \mathrm{~min}$.

\section{Analyse pollinique des échantillons de miel}

L'analyse pollinique a été réalisée selon la méthode établie par la Commission de botanique apicole et décrite par Louveaux et al. (1978) ainsi que Von Der Ohe et al. (2004). Dix grammes de miel sont pesés et dissous dans $20 \mathrm{~mL}$ d'eau chaude ne dépassant pas $40{ }^{\circ} \mathrm{C}$. La solution obtenue est centrifugée pendant $5 \mathrm{~min}$. Le culot restant au fond du tube est repris par $10 \mathrm{~mL}$ d'eau distillée et centrifugé pendant $10 \mathrm{~min}$ à 3000 tours/min puis transféré sur une lame porte-objet, séché et identifié. L'identification des grains de pollen a été établie grâce aux banques de données numériques et bibliographiques du laboratoire d'analyse et d'écologie apicole du Centre d'études techniques apicole de Moselle (Cetam) en Lorraine. Les préparations ont été explorées à différents grossissements $(\times 100, \times 400$ et $\times 1$ 000). Les pollens des plantes anémophiles ou de plantes dépourvues de nectar sont à retrancher du total des pollens dénombrés avant que ne soient calculés les pourcentages des pollens de plantes nectarifères. La fréquence en pourcentage des types de pollen est établie après le dénombrement d'au moins 300 grains de pollen (FellerDemasly et al., 1989). Dans l'estimation des fréquences des différents pollens, les termes suivants sont utilisés :

- pollens dominants, pour les formes qui représentent plus de $45 \%$ de pollen dénombré ;
- pollens secondaires, pour les grains de pollen qui ont une fréquence comprise entre 16 et $45 \%$

- pollens tertiaires, pour les grains de pollen qui ont une fréquence comprise entre 3 et $15 \%$

- pollens rares ou isolés, pour les grains de pollen qui ont une fréquence inférieure à $3 \%$.

Un miel est considéré monofloral lorsque le nombre de pollens dominants provenant d'une espèce de fleur est supérieur ou égal à $45 \%$.

\section{Résultats et discussion}

\section{Analyses physico-chimiques}

Les résultats des analyses physicochimiques sont résumés au tableau 1. La conductivité électrique dépend de la teneur en éléments minéraux et de l'acidité du miel : plus ces dernières sont élevées et plus la conductivité correspondante est élevée. Il existe une relation linéaire entre ces grandeurs mesurées (Piazza et al., 1991). Les miels des régions étudiées présentent des conductivités électriques variant entre $0,20 \mathrm{mS} / \mathrm{cm}$ $\pm 0,01$ (Ba5) et $0,80 \mathrm{mS} / \mathrm{cm} \pm 0,06$ (A1). Les échantillons A1 et Bs2 sont des miels monofloraux constitués respectivement de 97 et $99 \%$ de pollen d'Eucalyptus (tableau 2) et présentent des conductivités électriques variant de $0,80 \mathrm{mS} / \mathrm{cm}$ $\pm 0,06$ à $0,76 \mathrm{mS} / \mathrm{cm} \pm 0,05$ respectivement. Pour ce type de miel, Talpay (1985) rapporte que la conductivité électrique est plus facilement mesurable. Elle est utilisée principalement pour la caractérisation de certains miels monofloraux. Il propose les valeurs de 0,40 à $0,90 \mathrm{mS} / \mathrm{cm}$ pour les miels d'Eucalyptus.

La connaissance de la teneur en eau est une donnée très importante pour la durée de vie du miel pendant l'entreposage (Terrab et al., 2003). Le miel qui contient une teneur élevée en eau fermente facilement. Les valeurs obtenues varient entre $14,40 \% \pm 0,22$ (T5) et 18,60\% $\%$ 0,28 (T7) et sont largement en dessous de la limite maximale préconisée par les normes européennes (Conseil de l'Union européenne, 2002) qui reprennent tout simplement les normes du Codex Alimentarius datant de 1981 mais révisées en 2001. Ce sont donc des normes internationales.
Les valeurs de $\mathrm{pH}$ des miels étudiés sont comprises entre 3,66 0,02 (Bs4) et 4,23 $\pm 0,01$ (A5). L'acidité totale est un critère très important. Nos échantillons présentent une faible acidité totale comprise entre $12,10 \pm 0,07 \mathrm{meq} / \mathrm{kg}$ et 50,40 $\pm 2,67 \mathrm{meq} / \mathrm{kg}$. Les valeurs obtenues pour l'hydroxyméthylfurfural (HMF) se situent entre $0,80 \pm 0,6 \mathrm{mg} / \mathrm{kg}$ (A7) et $38,70 \pm 1,8 \mathrm{mg} / \mathrm{kg}$ (Ba2). Les recommandations de l'Union européenne (2002) fixent un maximum de $40 \mathrm{mg}$ d'HMF/ $\mathrm{kg}$ de miel.

L'activité diastasique des échantillons de miel étudiés varie entre 8,00 unités de Schade/g $\pm 0,1$ et 34,00 unités de $\mathrm{Schade} / \mathrm{g} \pm 1,2$. Nous sommes en présence de miels frais, de qualité conformes aux normes. Selon Terrab et al. (2001) et Nagai et al. (2002), les glucides des miels sont essentiellement des monosaccharides réducteurs tels que le glucose et le fructose qui représentent à eux seuls $90 \%$ de la matière sèche totale du miel (Gonnet, 1982). La teneur en glucose des 28 échantillons varie entre 27,20\% $\pm 0,72$ (Bs7) et $37,40 \% \pm 1,02$ (A5) ; celle du fructose est comprise entre $36,70 \% \pm 0,2 \quad$ (T2) et $45,90 \% \pm 0,78$ (Ba3). Ces résultats concordent avec ceux établis par Gonnet (1979) qui précise que la teneur des miels varie de 25,50 à $40,80 \%$ pour le glucose et de 32,40 à $45,90 \%$ pour le fructose. Le fructose prédomine dans presque tous les types de miels (White, 1979). Cette constatation est vérifiée pour tous les miels des régions du Nord algérien, sauf pour l'échantillon A5 qui est un miel de Brassicaceae (tableau 2). À ce sujet, Louveaux (1968) précise que la composition en sucres permet dans certains cas d'identifier l'origine botanique de quelques miels monofloraux tels que les miels de Brassicaceae caractérisés par une dominance du glucose par rapport au fructose. Les taux de saccharose des miels des régions étudiées varient de 0 à 7,6\% $\pm 0,08$. Tous nos échantillons correspondent aux normes établies par Bocquet (1997) qui fixe une limite maximale de $10 \%$ de saccharose.

\section{Analyses polliniques}

Les tableaux 2 et 3 mettent en évidence la dominance de cinq types de miel, à savoir des miels de Citrus (échantillons T5, T6, A4, A7, Bs1, Bs3, Ba3, Ba5 et Ba7), des miels polyfloraux (échantillons T2, T7, Bs4, Bs5, Bs6, Bs7, Ba1et Ba4), des miels d'Eucalyptus (échantillons T3, T4, A1, A3, 
Tableau 1. Résultats des analyses physico-chimiques des échantillons de miel.

Table 1. Results of the physico-chemical analyses of honey samples.

\begin{tabular}{|c|c|c|c|c|c|c|c|c|c|}
\hline $\begin{array}{l}\text { Échantil- } \\
\text { lon }\end{array}$ & $\begin{array}{c}\text { Conductivité } \\
\text { électrique } \\
\text { (mS/cm) }\end{array}$ & $\begin{array}{c}\text { Teneur en eau } \\
(\%)\end{array}$ & $\underset{\text { initial }}{\mathrm{pH}}$ & $\begin{array}{c}\text { Acidité } \\
\text { totale } \\
\text { (meq/kg) }\end{array}$ & $\begin{array}{l}\text { Hydroxy- } \\
\text { méthylfurfural } \\
\text { (mg/kg) }\end{array}$ & $\begin{array}{l}\text { Activité } \\
\text { diastasique } \\
\text { (unité de } \\
\text { Schade/g) }\end{array}$ & $\begin{array}{c}\text { Glucose } \\
(\%)\end{array}$ & $\begin{array}{c}\text { Fructose } \\
(\%)\end{array}$ & $\begin{array}{c}\text { Saccharose } \\
(\%)\end{array}$ \\
\hline $\mathrm{T} 1$ & $0,28 \pm 0,02$ & $14,5 \pm 0,24$ & $3,76 \pm 0,04$ & $40,6 \pm 2,01$ & $12,5 \pm 0,6$ & $30 \pm 1,4$ & $32,3 \pm 1,28$ & $37,4 \pm 0,87$ & $1,8 \pm 0,01$ \\
\hline $\mathrm{T} 2$ & $0,22 \pm 0,01$ & $14,8 \pm 0,22$ & $3,77 \pm 0,04$ & $25,5 \pm 1,01$ & $10,9 \pm 0,5$ & $11 \pm 0,2$ & $29,1 \pm 0,58$ & $36,7 \pm 0,20$ & $1,2 \pm 0,03$ \\
\hline T3 & $0,61 \pm 0,04$ & $14,8 \pm 0,24$ & $3,75 \pm 0,01$ & $34,4 \pm 1,08$ & $7,3 \pm 0,5$ & $19 \pm 0,6$ & $32,9 \pm 0,63$ & $39,3 \pm 0,76$ & $3,7 \pm 0,02$ \\
\hline $\mathrm{T} 4$ & $0,55 \pm 0,02$ & $14,8 \pm 0,16$ & $3,82 \pm 0,02$ & $40,6 \pm 1,37$ & $28,1 \pm 1,3$ & $19 \pm 0,4$ & $31,8 \pm 0,43$ & $39,9 \pm 1,32$ & $1,7 \pm 0,05$ \\
\hline T5 & $0,23 \pm 0,01$ & $14,4 \pm 0,22$ & $3,82 \pm 0,05$ & $25,1 \pm 0,76$ & $10,9 \pm 1,2$ & $13 \pm 0,9$ & $35,9 \pm 0,38$ & $44,5 \pm 0,39$ & $1,3 \pm 0,03$ \\
\hline T6 & $0,28 \pm 0,01$ & $14,5 \pm 0,10$ & $3,78 \pm 0,04$ & $28,0 \pm 0,93$ & $16,4 \pm 0,8$ & $10 \pm 0,5$ & $32,1 \pm 0,45$ & $41,7 \pm 0,83$ & $1,3 \pm 0,02$ \\
\hline T7 & $0,22 \pm 0,02$ & $18,6 \pm 0,28$ & $3,77 \pm 0,05$ & $26,6 \pm 1,12$ & $10,3 \pm 0,2$ & $13 \pm 0,2$ & $28,4 \pm 0,79$ & $36,8 \pm 0,67$ & $1,3 \pm 0,02$ \\
\hline A1 & $0,80 \pm 0,06$ & $15,0 \pm 0,26$ & $3,94 \pm 0,04$ & $36,7 \pm 1,09$ & $9,2 \pm 0,4$ & $13 \pm 0,3$ & $31,3 \pm 0,44$ & $37,9 \pm 0,16$ & $7,6 \pm 0,08$ \\
\hline $\mathrm{A} 2$ & $0,52 \pm 0,02$ & $14,8 \pm 0,32$ & $3,90 \pm 0,05$ & $44,1 \pm 2,06$ & $12,5 \pm 0,5$ & $34 \pm 1,2$ & $31,9 \pm 0,54$ & $43,9 \pm 0,66$ & $1,1 \pm 0,01$ \\
\hline A3 & $0,41 \pm 0,02$ & $14,8 \pm 0,22$ & $3,89 \pm 0,05$ & $15,5 \pm 0,24$ & $22,5 \pm 0,9$ & $8 \pm 0,2$ & $30,9 \pm 0,65$ & $44,4 \pm 0,66$ & $1,1 \pm 0,01$ \\
\hline A4 & $0,22 \pm 0,02$ & $14,6 \pm 0,22$ & $3,83 \pm 0,02$ & $22,8 \pm 0,84$ & $3,7 \pm 0,1$ & $10 \pm 0,5$ & $33,3 \pm 1,35$ & $42,6 \pm 0,38$ & $1,4 \pm 0,02$ \\
\hline A5 & $0,25 \pm 0,02$ & $14,5 \pm 0,28$ & $4,23 \pm 0,01$ & $12,1 \pm 0,07$ & $24,3 \pm 1,5$ & $18 \pm 0,8$ & $37,4 \pm 1,02$ & $36,7 \pm 0,84$ & $1,4 \pm 0,01$ \\
\hline A6 & $0,63 \pm 0,03$ & $14,7 \pm 0,26$ & $3,79 \pm 0,03$ & $34,8 \pm 1,09$ & $9,8 \pm 0,6$ & $8 \pm 0,1$ & $31,8 \pm 0,43$ & $36,7 \pm 0,63$ & $5,6 \pm 0,20$ \\
\hline A7 & $0,21 \pm 0,01$ & $18,4 \pm 0,42$ & $3,73 \pm 0,03$ & $25,6 \pm 0,75$ & $0,8 \pm 0,6$ & $8 \pm 0,3$ & $31,3 \pm 0,56$ & $41,0 \pm 0,67$ & $1,1 \pm 0,01$ \\
\hline Bs1 & $0,24 \pm 0,02$ & $14,8 \pm 0,22$ & $3,81 \pm 0,05$ & $28,8 \pm 0,62$ & $12,5 \pm 0,9$ & $12 \pm 0,5$ & $31,0 \pm 0,59$ & $40,0 \pm 0,68$ & $1,4 \pm 0,03$ \\
\hline Bs2 & $0,76 \pm 0,05$ & $15,0 \pm 0,24$ & $3,81 \pm 0,03$ & $41,1 \pm 2,10$ & $18,4 \pm 0,8$ & $22 \pm 0,9$ & $31,9 \pm 0,36$ & $43,9 \pm 0,87$ & $1,1 \pm 0,01$ \\
\hline Bs3 & $0,55 \pm 0,04$ & $14,5 \pm 0,28$ & $3,83 \pm 0,03$ & $23,0 \pm 1,15$ & $13,9 \pm 0,9$ & $11 \pm 0,3$ & $33,0 \pm 0,63$ & $41,7 \pm 1,03$ & $1,0 \pm 0,00$ \\
\hline Bs4 & $0,67 \pm 0,05$ & $18,0 \pm 0,52$ & $3,66 \pm 0,02$ & $40,0 \pm 1,4$ & $21,5 \pm 1,6$ & $14 \pm 0,2$ & $32,9 \pm 0,32$ & $38,6 \pm 0,76$ & $3,3 \pm 0,05$ \\
\hline Bs5 & $0,24 \pm 0,01$ & $14,6 \pm 0,40$ & $4,21 \pm 0,01$ & $25,1 \pm 0,92$ & $6,2 \pm 0,6$ & $18 \pm 0,7$ & $31,6 \pm 0,70$ & $43,2 \pm 0,67$ & $1,6 \pm 0,01$ \\
\hline Bs6 & $0,22 \pm 0,01$ & $14,6 \pm 0,32$ & $3,85 \pm 0,04$ & $23,2 \pm 1,03$ & $8,0 \pm 0,4$ & $10 \pm 0,2$ & $31,4 \pm 0,45$ & $39,8 \pm 0,48$ & $1,4 \pm 0,05$ \\
\hline Bs7 & $0,54 \pm 0,03$ & $14,6 \pm 0,16$ & $3,95 \pm 0,05$ & $47,4 \pm 0,89$ & $9,7 \pm 0,5$ & $34 \pm 1,4$ & $27,2 \pm 0,72$ & $37,0 \pm 1,28$ & $1,2 \pm 0,03$ \\
\hline Ba1 & $0,27 \pm 0,01$ & $18,5 \pm 0,42$ & $3,78 \pm 0,04$ & $29,1 \pm 0,89$ & $15,3 \pm 0,6$ & $10 \pm 0,1$ & $31,7 \pm 0,35$ & $40,0 \pm 0,67$ & $1,0 \pm 0,01$ \\
\hline $\mathrm{Ba} 2$ & $0,52 \pm 0,03$ & $15,0 \pm 0,22$ & $3,75 \pm 0,01$ & $39,4 \pm 0,46$ & $38,7 \pm 1,8$ & $23 \pm 0,8$ & $32,8 \pm 0,79$ & $41,1 \pm 0,86$ & 0,0 \\
\hline Ba3 & $0,21 \pm 0,02$ & $14,6 \pm 0,23$ & $3,82 \pm 0,03$ & $22,0 \pm 0,32$ & $12,0 \pm 0,2$ & $25 \pm 1,3$ & $32,7 \pm 0,87$ & $45,9 \pm 0,78$ & $1,3 \pm 0,02$ \\
\hline $\mathrm{Ba} 4$ & $0,51 \pm 0,03$ & $15,0 \pm 0,14$ & $3,79 \pm 0,03$ & $50,4 \pm 2,67$ & $8,7 \pm 0,8$ & $20 \pm 0,9$ & $32,0 \pm 0,32$ & $39,5 \pm 0,73$ & $1,5 \pm 0,03$ \\
\hline Ba5 & $0,20 \pm 0,01$ & $14,4 \pm 0,42$ & $3,93 \pm 0,02$ & $15,9 \pm 0,40$ & $11,1 \pm 0,9$ & $8 \pm 0,1$ & $31,1 \pm 0,66$ & $40,0 \pm 0,46$ & $2,8 \pm 0,04$ \\
\hline Ba6 & $0,55 \pm 0,04$ & $15,0 \pm 0,20$ & $3,83 \pm 0,04$ & $28,4 \pm 0,59$ & $11,1 \pm 0,5$ & $15 \pm 0,8$ & $31,0 \pm 0,86$ & $38,6 \pm 0,38$ & $1,9 \pm 0,06$ \\
\hline $\mathrm{Ba} 7$ & $0,22 \pm 0,02$ & $18,4 \pm 0,40$ & $3,86 \pm 0,04$ & $20,8 \pm 0,39$ & $38,7 \pm 1,2$ & $14 \pm 0,5$ & $33,2 \pm 0,78$ & $40,9 \pm 1,33$ & $1,6 \pm 0,05$ \\
\hline Normes & $\leq 0,80$ & $\leq 20$ & $3,3-4,6$ & - & $\leq \mathbf{4 0}$ & $\geq 8$ & $25,5-40,8$ & $32,4-45,9$ & 10 \\
\hline
\end{tabular}

* Les valeurs représentent la moyenne de trois répétitions \pm écart type. 
Tableau 2. Pollens dominants, secondaires et tertiaires présents dans les échantillons de miel (en pourcentage).

Table 2. Dominant pollens, secondary pollens and minor pollens present in honey samples (in percentage).

\begin{tabular}{|c|c|c|c|}
\hline Échantillons & $\begin{array}{l}\text { Pollens dominants } \\
\qquad(\geq 45 \%)\end{array}$ & $\begin{array}{l}\text { Pollens secondaires } \\
(\geq 16 \% \text { et }<45 \%)\end{array}$ & $\begin{array}{l}\text { Pollens tertiaires } \\
(\geq 3 \% \text { et }<16 \%)\end{array}$ \\
\hline T1 & Hedysarum coronarium (95) & (0) & $(0)$ \\
\hline $\mathrm{T} 2$ & (0) & $\begin{array}{l}\text { Eucalyptus (30), Echium (23), } \\
\text { Citrus (20) }\end{array}$ & $\begin{array}{l}\text { Brassicaceæ (3), Trifolium repens (3), } \\
\text { Prunus/Pyrus (3) }\end{array}$ \\
\hline T3 & Eucalyptus (92) & $(0)$ & Apiaceæ (3) \\
\hline T4 & Eucalyptus (80) & (0) & Trifolium sp. (4), Echium (6) \\
\hline T5 & (0) & Citrus sp. (44) & $\begin{array}{l}\text { Brassicaceæ (9), Fabaceæ (8), } \\
\text { Trifolium sp. (6) }\end{array}$ \\
\hline T6 & (0) & Citrus sp. (30) & $\begin{array}{l}\text { Castanea sativa (13), Eucalyptus (10), } \\
\text { Echium (6), Prunus/Pyrus (4) }\end{array}$ \\
\hline T7 & (0) & Apiaceæ (26), Lotus (25) & Eucalyptus (6) \\
\hline A1 & Eucalyptus (97) & $(0)$ & $(0)$ \\
\hline A2 & Hedysarum coronarium (54) & Apiaceæ (21) & $\begin{array}{l}\text { Trifolium sp. (4), } \\
\text { Asteraceæ liguliforme (4) }\end{array}$ \\
\hline A3 & Eucalyptus (87) & $(0)$ & Prunus/Pyrus (3) \\
\hline A4 & (0) & Citrus sp. (33), Prunus/Pyrus (28) & $\begin{array}{l}\text { Brassicaceæ (5), Fabaceæ (8), } \\
\text { Carduus sp. (4), Borago (3) }\end{array}$ \\
\hline A5 & Brassicaceae (53) & Eucalyptus (22) & Lamiaceæ (8), Fabaceæ (7) \\
\hline A6 & Eucalyptus (79) & Apiaceæ (18) & (0) \\
\hline A7 & Citrus sp. (50) & (0) & $(0)$ \\
\hline Bs1 & (0) & Citrus sp. (34), Echium (18) & $\begin{array}{l}\text { Brassicaceæ (11), Fabaceæ (7), } \\
\text { Prunus/Pyrus (5) }\end{array}$ \\
\hline Bs2 & Eucalyptus (99) & $(0)$ & (0) \\
\hline Bs3 & (0) & Citrus sp. (32), Brassicaceæ (29) & Lamiaceæ (5) \\
\hline Bs4 & (0) & Echium (44), Eucalyptus (42) & Brassicaceæ (4), Trifolium sp. (8) \\
\hline Bs5 & (0) & $\begin{array}{l}\text { Apiaceæ (40), Trifolium sp. (22), } \\
\text { Eucalyptus (18) }\end{array}$ & Lotus (4) \\
\hline Bs6 & (0) & $\begin{array}{l}\text { Citrus sp. (21), Brassicaceæ (21), } \\
\text { Prunus/Pyrus (18) }\end{array}$ & (0) \\
\hline Bs7 & (0) & (0) & $\begin{array}{l}\text { Eucalyptus (15), Zizyphus sp. (13), } \\
\text { Brassicaceæ (4) }\end{array}$ \\
\hline Ba1 & (0) & Brassicaceæ (41), Trifolium sp. (18) & $(0)$ \\
\hline $\mathrm{Ba} 2$ & Eucalyptus (74) & (0) & Apiaceæ (4), Lamiaceæ (8), Citrus (5) \\
\hline Ba3 & Citrus sp. (50) & Echium (16) & Fabaceæ (6) \\
\hline $\mathrm{Ba} 4$ & (0) & Daucus (39) & $\begin{array}{l}\text { Brassicaceæ (5), Fabaceæ (5), } \\
\text { Carduus sp. (3), Ulariaceæ (5), Citrus (4), } \\
\text { Hedysarum coronarium (3) } \\
\text { Eucalyptus (4), Prunus/Pyrus (4) }\end{array}$ \\
\hline Ba5 & Citrus sp. (69) & $(0)$ & Brassicaceæ (14), Lamiaceæ (5) \\
\hline Ba6 & Eucalyptus (90) & $(0)$ & $(0)$ \\
\hline $\mathrm{Ba} 7$ & Citrus sp. (59) & $(0)$ & Prunus/Pyrus (15), Fabaceæ (10) \\
\hline
\end{tabular}

$(0)=$ pas de pollen $; n=300$ grains de pollen.

A6, Bs2, Ba2 et Ba6), des miels d'Hedysarum coronarium (échantillons T1 et A2) et de Brassicaceae (échantillon A5). Malgré le nombre restreint de formes de dominance, plusieurs espèces sont identifiées comme pollens secondaires, tertiaires ou rares (tableaux 2 et 3). Il s'agit du pollen d'Echium, de Prunus/Pyrus,
d'Apiaceae, de Lotus, de Trifolium sp., de Daucus, d'Asteraceae, de Lamiaceae et de Fabaceae, caractérisant la flore méditerranéenne. Selon Ricciardelli d'Albore (1998), les Citrus, l'Eucalyptus et le Trifolium constituent les principales espèces mellifères en Algérie. Les miels monofloraux obtenus de pollen de Citrus constituent la partie la plus importante des miels étudiés des régions du Nord (9/28). Le pollen d'Eucalyptus constitue aussi une source importante pour les miels étudiés : il est omniprésent dans plus de $71 \%$ des échantillons. Son pourcentage atteint même 99\% dans l'échantillon Bs2. Cette forte présence est liée à la taille 
Tableau 3. Les différents types de pollen présents dans les échantillons de miel (en pourcentage).

Table 3. The various types of pollen found in honey samples (in percentage).

\begin{tabular}{|c|c|}
\hline Échantillons & Pollens rares ou isolés ( $<3 \%)$ \\
\hline $\mathrm{T} 1$ & $\begin{array}{l}\text { Geranium sp., Centaurea sp., Asteraceæ liguliforme, Taraxacum, Brassicaceæ, Lamiaceæ, Carduus sp., } \\
\text { Prunus/Pyrus, Trifolium sp. }\end{array}$ \\
\hline $\mathrm{T} 2$ & Apiaceæ, Anthyllis, Erica arborea, Medicago, Lamiaceæ, Carduus sp. \\
\hline T3 & Trifolium sp., Citrus, Hedysarum coronarium, Brassicaceæ, Carduus sp., Acacia sp., Convolvulus \\
\hline T4 & Asteraceæ liguliforme, Citrus, Brassicaceæ, Alliaceæ, Capparis, Borago, Convolvulus, Cirsium \\
\hline T5 & Echium, Prunus/Pyrus, Apiaceæ, Eucalyptus, Acacia sp., Euphorbiaceæ, Convolvulus, Carduus sp. \\
\hline T6 & Cirsium, Achillea type, Ceratonia siliqua, Carduus sp., Brassicaceæ \\
\hline T7 & Cynoglossum, Lamiaceæ, Brassicaceæ \\
\hline A1 & Fabaceæ, Citrus, Apiaceæ, Carduus sp., Asteraceæ liguliforme, Trifolium sp. \\
\hline A2 & $\begin{array}{l}\text { Mentha, Lamiaceæ, Eucalyptus, Achillea type, Ceratonia ciliqua, Echinops, Rhamnaceæ, Onobrychis, } \\
\text { Carduus sp., Convolvulus, Cirsium }\end{array}$ \\
\hline A3 & $\begin{array}{l}\text { Brassicaceæ, Citrus, Genista type, Zizyphus, Hédysarum coronarium, Fabaceæ, Liliaceæ, Convolvulus, } \\
\text { Cirsium }\end{array}$ \\
\hline A4 & Lamiaceæ, Apiaceæ, Trifolium repens, Acacia sp. \\
\hline A5 & $\begin{array}{l}\text { Scrofulariaceæ, Trifolium sp., Solidago type, Apiaceæ, Carduus sp., Acacia sp., Astéraceæ liguliforme, } \\
\text { Liliaceæ }\end{array}$ \\
\hline A6 & Citrus, Echium, Trifolium sp. \\
\hline A7 & Echium, Brassicaceæ, Eucalyptus \\
\hline Bs1 & Lamiaceæ, Carduus sp., Castanea, Erica arborea, Taraxacum, Trifolium repens, Eucalyptus, Convolvulus \\
\hline Bs2 & Capparis, Cirsium, Trifolium sp., Convolvulus, Apiaceæ, Acacia sp., Astéraceæ liguliforme \\
\hline Bs3 & Cynoglossum, Prunus/Pyrus, Fabaceæ, Rosaceæ, Convolvulus, Apiaceæ, Carduus sp., Genista type, Cirsium \\
\hline Bs4 & Taraxacum, Capparis, Astéraceæ liguliforme, Acacia sp., Convolvulus, Vicia, Prunus/Pyrus \\
\hline Bs5 & Citrus, Fabaceæ, Carduus sp., Asteraceæ liguliforme, Rhamnaceæ \\
\hline Bs6 & Apiaceæ, Salix, Lotus \\
\hline Bs7 & Carduus sp., Echium, Rubus type, Genista type, Lamiaceæ, Achillea type, Centaurea, Echinops \\
\hline Ba1 & Citrus, Echium, Salix, Hedysarum coronarium, Myrtus communis, Trifolium repens, Lamiaceæ, Eucalyptus \\
\hline $\mathrm{Ba} 2$ & Carduus sp., Echium, Asteraceæ liguliforme, Acacia sp., Malva sp., Prunus/pyrus, Convolvulus \\
\hline Ba3 & Brassicaceæ, Carduus sp., Prunus/pyrus, Trifolium sp., Acacia sp., Convolvulus \\
\hline $\mathrm{Ba} 4$ & Arctium type, Convolvulus, Trifolium sp., Apiaceæ, Genista type, Cucumis \\
\hline Ba5 & Rhamnaceæ, Eucalyptus, Trifolium sp., Malvaceæ \\
\hline Ba6 & Brassicaceæ, Citrus, Apiaceæ, Convolvulus, Trifolium sp., Lamiaceæ, Ceratonia siliqua \\
\hline Ba7 & Malvaceæ, Trifolium sp., Brassicaceæ, Carduus sp., Eucalyptus, Lotus \\
\hline
\end{tabular}

(entre 25 et $35 \mu$ ) ainsi qu'à l'abondance de pollen dans les fleurs de Myrtaceae. Ainsi l'Eucalyptus constitue une plante apicole très intéressante en Mitidja.

Le pollen d'Hedysarum coronarium (Sainfoin ou Sulla) domine dans deux échantillons de miel (échantillons T1 et A2). La présence de ce pollen s'explique par l'importance de la culture de cette espèce dans ces régions. Louveaux et Abed (1984) soulignent que l'Hedysarum coronarium est dominant dans la partie centrale de l'Algérie, en Tunisie et au Maroc, ce qui concorde avec nos résultats. Nous avons mis en évidence un seul miel monofloral de Brassicaceae (échantillon A5) dans la région d'Alger. Les miels polyfloraux sont en nombre appréciable
(8/28) et sont constitués à partir de pollens d'origines diverses (plantes spontanées, arbres fruitiers ou autres) sans dominance apparente. Ces miels comportent des pollens secondaires $(\geq 16 \%$ et $<45 \%$ ) de un, deux ou trois taxons. Nous notons en particulier la présence de pollens de type Prunus/Pyrus dans plusieurs échantillons (8/28), pollens des différentes espèces fruitières de rosacées issus des vergers nombreux en Mitidja. Les pollens rares (tableau 3) proviennent d'une grande diversité de plantes spontanées ou cultivées reflétant la diversité botanique des régions du Nord de l'Algérie.

Les miels de Citrus et d'Eucalyptus sont présents dans les quatre régions d'étude.
Dans la préfecture de Blida dominent les miels de Citrus (pourcentages de pollens dominants 50,59 et $69 \%$ ). En effet, cette région est connue comme étant à vocation agrumicole. Dans les régions d'Alger et de Tipaza, les miels de Citrus contiennent des pollens secondaires (inférieurs à $45 \%$ ) en plus grande quantité par rapport à Blida. Dans ces deux régions prédominent les miels d'Eucalyptus avec des pourcentages de pollens dominants très élevés à savoir 97, 87 et $79 \%$ pour la région d'Alger et 92 et $80 \%$ pour la région de Tipaza. La région de Boumerdès est caractérisée par les miels polyfloraux et en moindre importance par des miels de Citrus dont le pourcentage de pollen est inférieur à $45 \%$ ainsi que par des miels d'Eucalyptus. 


\section{Conclusion}

La caractérisation de l'appellation miel monofloral est fondée à la fois sur des analyses physico-chimiques, polliniques et sensorielles. En général, les miels monofloraux possèdent un ou plusieurs caractères discriminants qui vont les différencier des autres miels monofloraux et des miels polyfloraux. Ces critères varient d'un miel à l'autre. Pour les miels d'Eucalyptus, c'est une conductivité électrique généralement assez élevée et un rapport fructose/glucose assez bas, expliqué par la forte teneur en glucose de ces miels. Les miels de Citrus possèdent en général une plus faible conductivité électrique et un rapport fructose/ glucose similaire à l'Eucalyptus.

La palynologie est plus difficile à interpréter car les pollens présents dépendent beaucoup de la flore annexe avec des artefacts liés à la sur- ou sousreprésentation de certains pollens. Elle est cependant importante pour établir la base d'un référentiel pollinique permettant de caractériser l'origine géographique du produit qui peut, dans quelques cas relativement rares, être reconnue grâce à des formes caractéristiques de pollen n'existant que sur un territoire déterminé. Le plus souvent, c'est l'apparition de combinaisons de pollens qui permet la localisation de la région où le miel a été produit. Les frontières de l'Algérie comme de tous autres pays sont des frontières politiques et non pas phytogéographiques. L'analyse pollinique pourra déterminer de façon certaine qu'un miel est originaire d'Afrique du Nord. Pour séparer les miels algériens des miels tunisiens ou marocains par exemple, il faudrait disposer d'analyses polliniques comparatives entre les miels de ces différents pays et surtout identifier des espèces mellifères spécifiques à chaque pays dont la présence de pollen dans le miel pourrait servir de marqueur.

Les résultats de ces analyses font apparâtre en Mitidja la dominance des miels monofloraux de Citrus, d'Eucalyptus et de miels polyfloraux qui ont une grande importance pour l'apiculture algérienne. Ces résultats constituent un complément de référentiel pour les miels étudiés des régions du Nord de l'Algérie et fournissent des éléments précis pour le contrôle des appellations des miels produits. Ils montrent également l'importance relative des différentes espèces nectarifères et permettent donc d'établir des fiches de spécifications qui pourront évoluer en fonction d'analyses d'autres miels.

À l'heure actuelle, l'utilisation de méthodes de contrôle fiables pour assurer la conformité d'un produit alimentaire est impérative pour limiter ou éliminer les risques de falsification. La recherche de miels falsifiés est fondée sur des analyses essentiellement physico-chimiques : l'adjonction de certains sirops de sucres dans les miels se traduit par un effet de dilution qui va diminuer la valeur de certains paramètres (enzymes, conductivité électrique), modifier les valeurs d'acidimétrie, et plus ou moins modifier le profil en sucres. Ces analyses permettent de mettre en évidence des adultérations.

\section{Références}

Anklam E. A review of analytical methods to determine the geographical and botanical origin of honey. Food Chem 1998 ; 61 : 549-62.

Anklam E, Radovic B. Suitable analytical methods for determining the origin of European honey. Am Laboratory 2001 ; (May) : 60-6.

Bocquet M. Le miel d'Eucalyptus. Nature et composition, principales caractéristiques organoleptiques. Bull Tech Apic $1997 ; 24: 151-2$.

Bogdanov S, Martin P, Lüllmann C. Harmonised methods of the European Honey Commission. Apidologie 1997 ; (extra issue) : 1-59.

Bogdanov S. Harmonized methods of the international honey commission. Commission duCodex Alimentarius. Norme régionale européenne recommandée pour le miel, 1984. Berne (Suisse) : FAO/OMS, 2002.

Devilliers J, Morlot M, Pham-Delegue MH, Dore J. C. Classification of monofloral honeys based on their quality control data. Food Chem 2004; $56: 305-31$.
Feller-Demasly MJ, Patent J. Analyse pollinique des miels de l'Ontario, Canada. Apidologie 1989 ; 20 : $127-38$.

Gonnet M. Quelques observations sur la production du nectar chez les lavandes et les lavandins en Provence. Apidologie $1979 ; 2$ : 303-8.

Gonnet M. Le miel, composition, propriétés et conservation. Echauffour (France): Ed. OPIDA, 1982.

Louveaux J, Abed L. Les miels d'Afrique du Nord et leur spectre pollinique. Apidologie $1984 ; 15$ : 145-70.

Louveaux J, Maurizio A, Vorwohl G. Methods of melissopalynology. Bee World 1978; 59 : 139-57.

Louveaux J. Composition, propriétés et technologie du miel. In: Chauvin R. Traité de biologie de l'abeille. Tome 3, les produits de la ruche. Paris: editions Masson, 1968

Mateo R, Bosch-Reig F. Sugars profiles of Spanish unifloral honeys. Food Chem 1997; 60 : 33-40.

Nagai T, Inoue R, Inoue H, Suzuki N. Scavenging capacities of pollen extracts from Cistus ladaniferus on autoxidation, superoxide radicals, hydroxyl radicals and DPPH radicals. Nut Res $2002 ; 22$ : 519-26.

Piazza MG, Accorti M, Persano Oddo L. Electrical conductivity, ash, colour and specific rotatory power in Italian unifloral honeys. Apicoltura $1991 ; 7: 51-63$

Popek SA. Procedure to identify honey type. Food Chem 2002; 79 : 401-6.

Ricciardelli d'Albore G. Mediterranean melisso palynology. Perugia (Italia) : Università degli. Studi, 1998

Talpay B. Spezifikationen Deutsch. LebensmRundsch $1985 ; 81: 148-51$

Terrab A, Vega-Pérez JM, Diez MJ, Heredia FJ. Characterisation of northwest Moroccan honeys by gas chromato-graphic-mass spectrometric analysis of their sugar components. J Sci Food Agric $2001 ; 82$ : 179-85.

Terrab A, Diez MJ, Heredia FJ. Characterisation of Moroccan unifloral honeys by their physicochemical characteristics. Food Chem 2002 : 79 337-73.

Terrab A, Diez MJ, Heredia FJ. Palynological, physicochemical and colour characterization of Moroccan honeys. Orange (Citrus sp.) honey. Int J Food Sci Technol 2003 ; 38 : 383-7.

Von der Ohe W, Persano Oddo L, Piana L, Morlot M, Martin P. Harmonised methods of melissopalynogie. Apidologie 2004 ; 35 : 18-25.

White JW. Spectrophotometric method for hydroxymethylfurfural in honey. J Asso Ana Chem $1979 ; 62$ : 509. 